\title{
Voice based email system for blinds
}

\author{
T.Shabana ${ }^{1}$, A.Anam², A.Rafiya ${ }^{3}$, K.Aisha ${ }^{4}$ \\ Assistant Professor, Computer Engineering, M.H. Saboo Siddik College of Engineering, Mumbai, India ${ }^{1}$ \\ UG Student, Computer Engineering, M.H. Saboo Siddik College of Engineering, Mumbai, India $2,3,4$
}

\begin{abstract}
In today's world communication has become so easy due to integration of communication technologies with internet. However the visually challenged people find it very difficult to utilize this technology because of the fact that using them requires visual perception. Even though many new advancements have been implemented to help them use the computers efficiently no naïve user who is visually challenged can use this technology as efficiently as a normal naïve user can do that is unlike normal users they require some practice for using the available technologies. This paper aims at developing an email system that will help even a naïve visually impaired person to use the services for communication without previous training. The system will not let the user make use of keyboard instead will work only on mouse operation and speech conversion to text. Also this system can be used by any normal person also for example the one who is not able to read. The system is completely based on interactive voice response which will make it user friendly and efficient to use.
\end{abstract}

Keywords: Visually challenged people, IVR, Speech to text converter, Mouse click event, Screen reader.

\section{INTRODUCTION}

Internet is considered as a major storehouse of information in today's world. No single work can be done without the help of it. It has even become one of the de facto methods used in communication. And out of all methods available email is one of the most common forms of communication especially in the business world. [4] However not all people can use the internet. This is because in order to access the internet you would need to know what is written on the screen. If that is not visible it is of no use.[2] This makes internet a completely useless technology for the visually impaired and illiterate people. Even the systems that are available currently like the screen readers TTS and ASR [1] do not provide full efficiency to the blind people so as to use the internet. As nearly 285 million people worldwide are estimated visually impaired it become necessary to make internet facilities for communication usable for them also.[5]

Therefore we have come up with this project in which we will be developing a voice based email system which will aid the visually impaired people who are naive to computer systems to use email facilities in a hassle free manner. The users of this system would not need to have any basic information regarding keyboard shortcuts or where the keys are located. All functions are based on simple mouse click operations making it very easy for any type of user to use this system.

Also the user need not worry about remembering which mouse click operation he/she needs to perform in order to avail a given service as the system itself will be prompting them as to which click will provide them with what operations.

\section{EXISTING SYSTEM}

There are a total number of 4.1 billion email accounts created until 2014 and an there will be estimated 5.2 billion accounts by end of 2018.[4] this makes emails the most used form of communication.
The most common mail services that we use in our day to day life cannot be used by visually challenged people. This is because they do not provide any facility so that the person in front can hear out the content of the screen. As they cannot visualize what is already present on screen they cannot make out where to click in order to perform the required operations.[3]

For a visually challenged person using a computer for the first time is not that convenient as it is for a normal user even though it is user friendly. Although there are many screen readers available then also these people face some minor difficulties. Screen readers read out whatever content is there on the screen and to perform those actions the person will have to use keyboard shortcuts as mouse location cannot be traced by the screen readers. This means two things; one that the user cannot make use of mouse pointer as it is completely inconvenient if the pointer location cannot be traced and second that user should be well versed with the keyboard as to where each and every key is located. A user is new to computer can therefore not use this service as they are not aware of the key locations.

Another drawback that sets in is that screen readers read out the content in sequential manner and therefore user can make out the contents of the screen only if they are in basic HTML format. Thus the new advanced web pages which do not follow this paradigm in order to make the website more user-friendly only create extra hassles for these people.

All these are some drawbacks of the current system which we will overcome in the system we are developing.

\section{PROPOSED SYSTEM}

The proposed system is based on a completely novel idea and is nowhere like the existing mail systems. The most important aspect that has been kept in mind while developing the proposed system is accessibility. A web system is said to be perfectly accessible only if it can be 
used efficiently by all types of people whether able or disable. The current systems do not provide this accessibility. Thus the system we are developing is completely different from the current system. Unlike current system which emphasizes more on user friendliness of normal users, our system focuses more on user friendliness of all types of people including normal people visually impaired people as well as illiterate people.

The complete system is based on IVR- interactive voice response. When using this system the computer will be prompting the user to perform specific operations to avail respective services and if the user needs to access the respective services then he/she needs to perform that operation.

One of the major advantages of this system is that user won't require to use the keyboard. All operations will be based on mouse click events. Now the question that arises is that how will the blind users find location of the mouse pointer.

As particular location cannot be tracked by the blind user the system has given the user a free will to click blandly anywhere on the screen. Which type of click will perform which function will be specified by the IVR. Thus user need not worry about location of the mouse at all.

This system will be perfectly accessible to all types of users as it is just based on simple mouse clicks and speech inputs and there is no need to remember keyboard shortcuts. Also because of IVR facility those who cannot read need not worry as they can listen to the prompting done by the system and perform respective actions.

\section{DESIGN}

A. User Interface Design:

The user interface is designed using Adobe Dreamweaver CS3. The complete website focuses more on efficiency in understanding the IVR rather than the look and feel of the system as the system is primarily developed for the blind people to whom the look and feel won't be of that primary importance as the efficiency of understanding the prompting would be.

\section{B. Database Design:}

Our system maintains a database for user validation and storing mails of the user. There are a total of five tables.

The relationship between them is assigned after much consideration. The E-R diagram of our complete system is depicted in Fig 1. The Inbox, Sent-Mail and Trash schemas will store all mails of the respective service that belongs to that particular user.

\section{System Design:}

Fig. 2 depicts the complete system design. It is the level-2 data flow diagram which gives complete detailed flow of events in the system. As we can see all operations are performed by mouse click events only. Also at some places voice input is required.

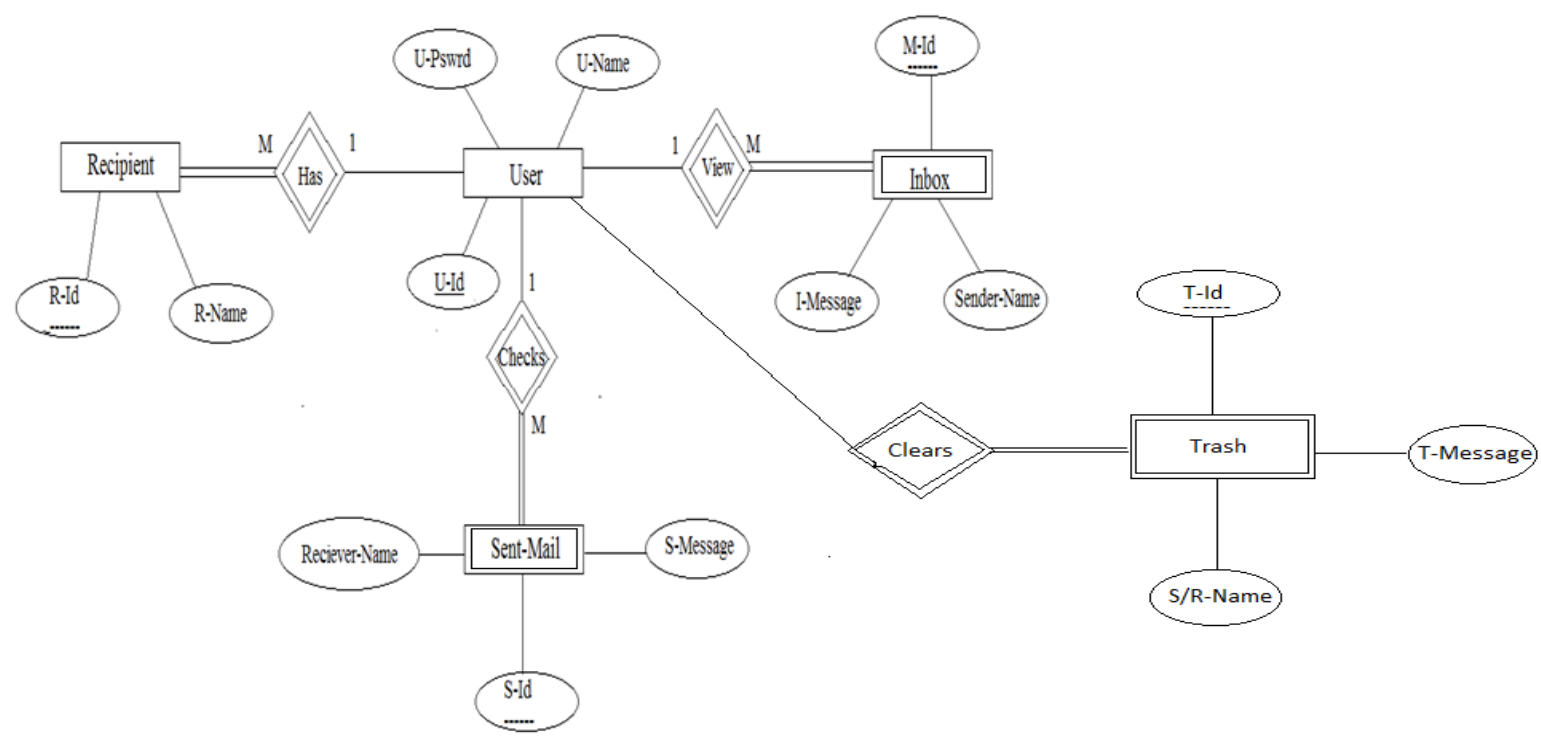

Fig. 1. E-R Diagram of our system 


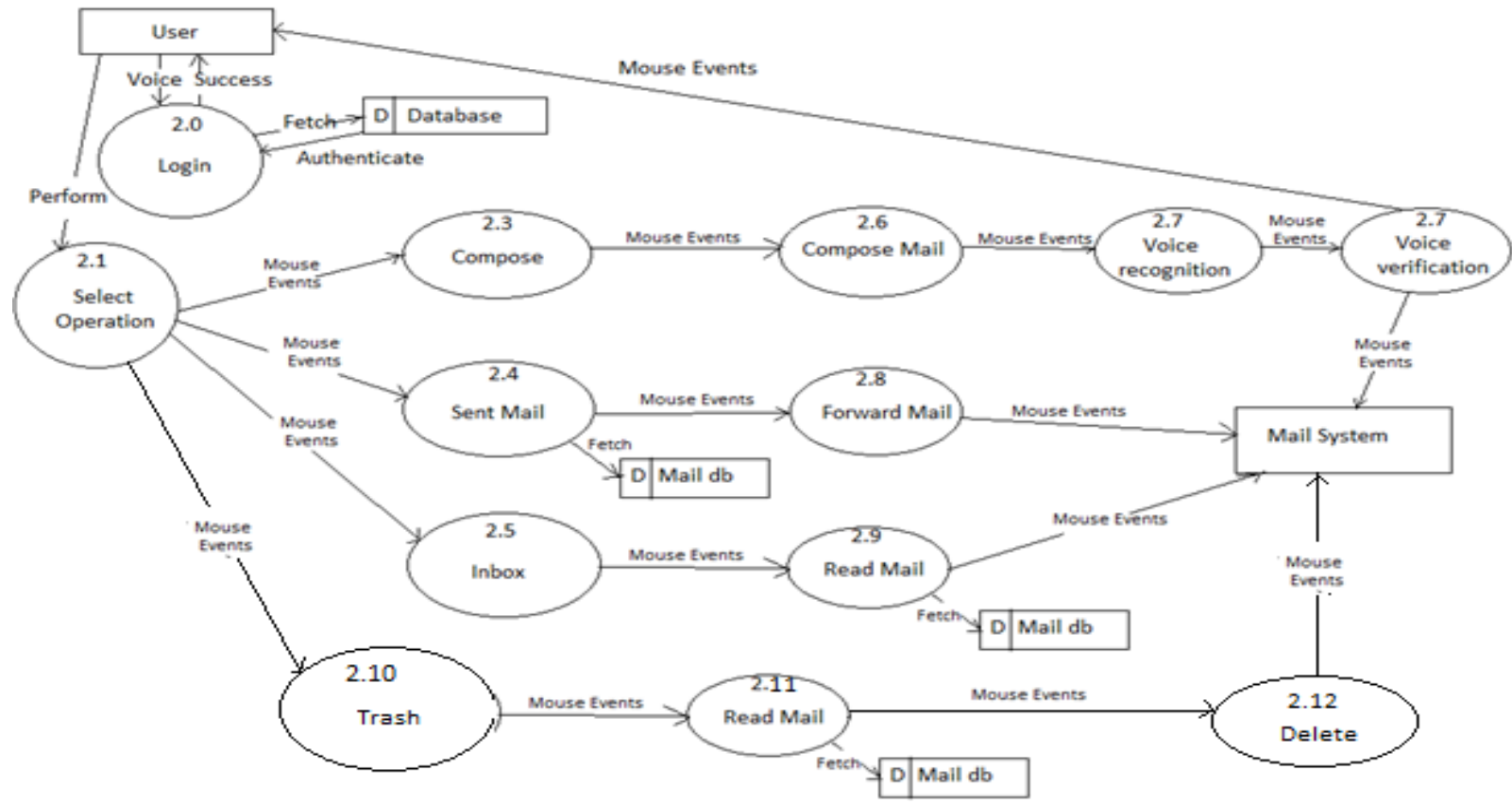

Fig. 1. Level-2 Data Flow Diagram of our system

\section{IMPLEMENTATION}

This system is currently being developed by us. The following are modules are the ones that are already developed. Their working is as follows:

\section{A. Registration:}

This is the first module of the system. Any user who wishes to use the system should first register to obtain username and password. This module will collect complete information of the user by prompting the user as to what details needs to be entered. The user will need to speak up the details to which the system will again confirm by prompting alphabetically. If the information is not correct user can re-enter else the prompt will specify the operation to be performed to confirm.

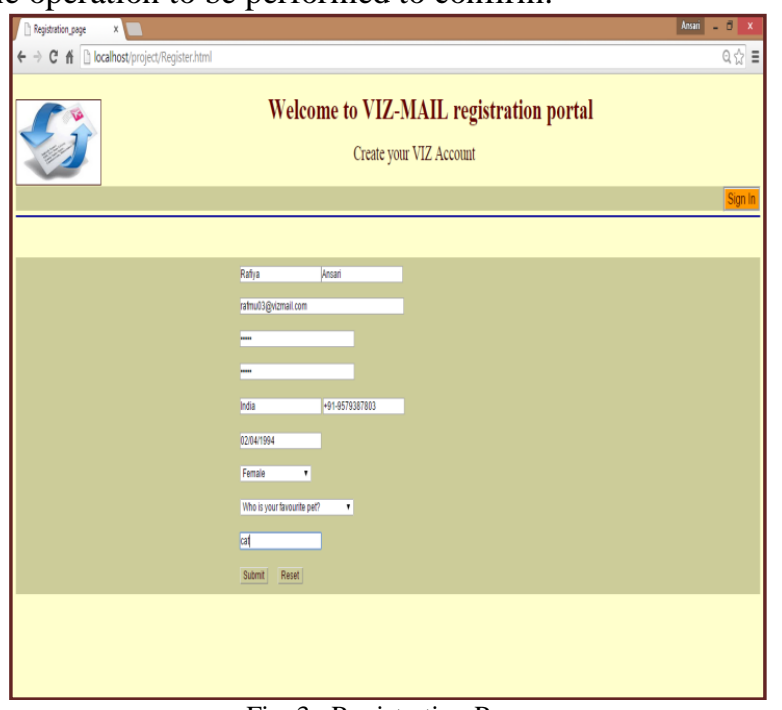

Fig. 3. Registration Page

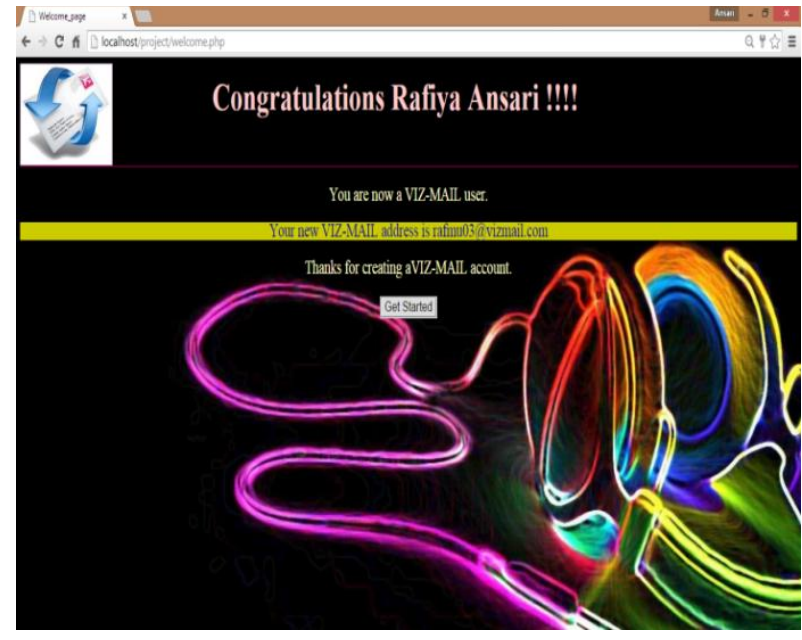

Fig. 4. Welcome Page

\section{B. Login:}

Once the registration is done the user can login to the system. This module will ask the user to provide the username and password. This will be accepted in speech. Speech conversion will be done to text and user will be told to validate whether the details are entered correctly or not. Once the entry is done correctly database will be checked for entry. If the user is authorized it will be directed to homepage. 
International Journal of Advanced Research in Computer and Communication Engineering

Vol. 4, Issue 1, January 2015

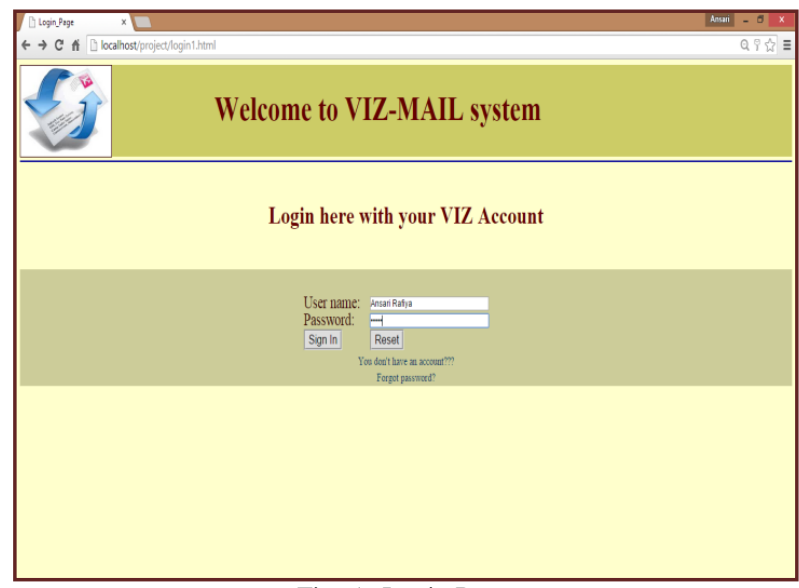

Fig. 5. Login Page

\section{Forgot Password:}

In case where an authorized user forgets the password and thus is not able to login he/she can select forgot password module. In this module the user will be first told to enter username. According to username the security question will be searched in database. This is the question provided at time of registration. The question will be spoken out by the computer. The user should in turn specify the answer that was provided by him/her during registration. If both get matched, user is given option to change password.
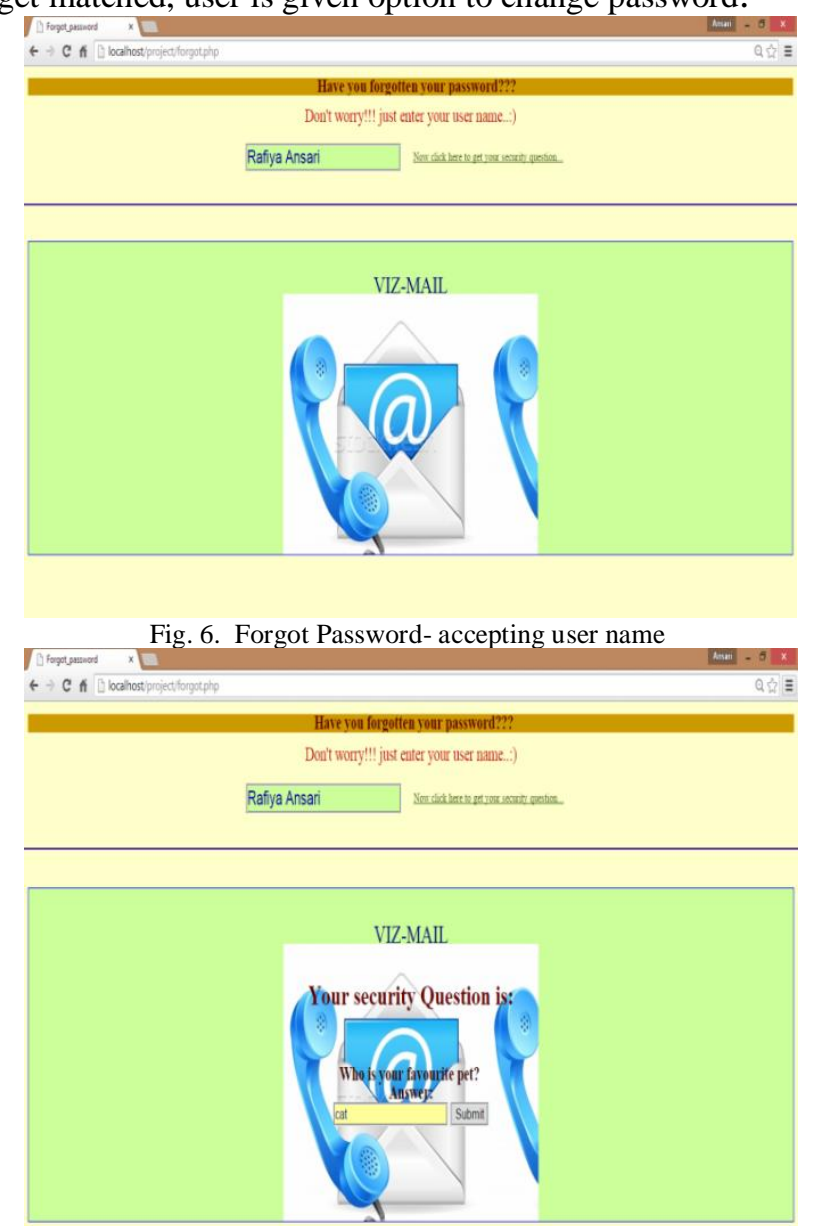

Fig. 7. Forgot password- Asking security question

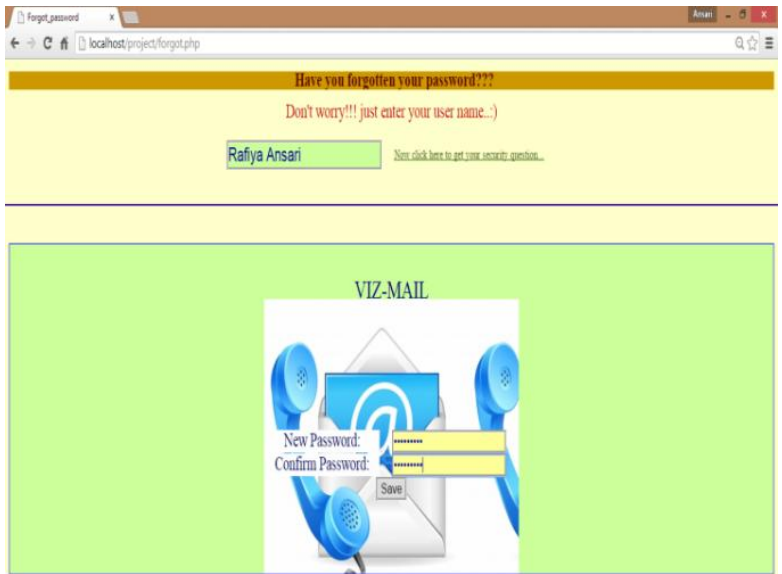

Fig. 8. Forgot Password- Asking new password

\section{Home Page:}

The user is redirected to this page once log in done successfully. From this page now the user can perform operations that the user wishes to perform. The options available are:

1. Inbox

2. Compose

3. Sent mail

4. Trash

Prompting will provide the mouse click operation that needs to be performed for the required service.

The double right click event is specifically reserved to log out of the system at any time the user wants to. This will be specified by the prompt right at the beginning after login.

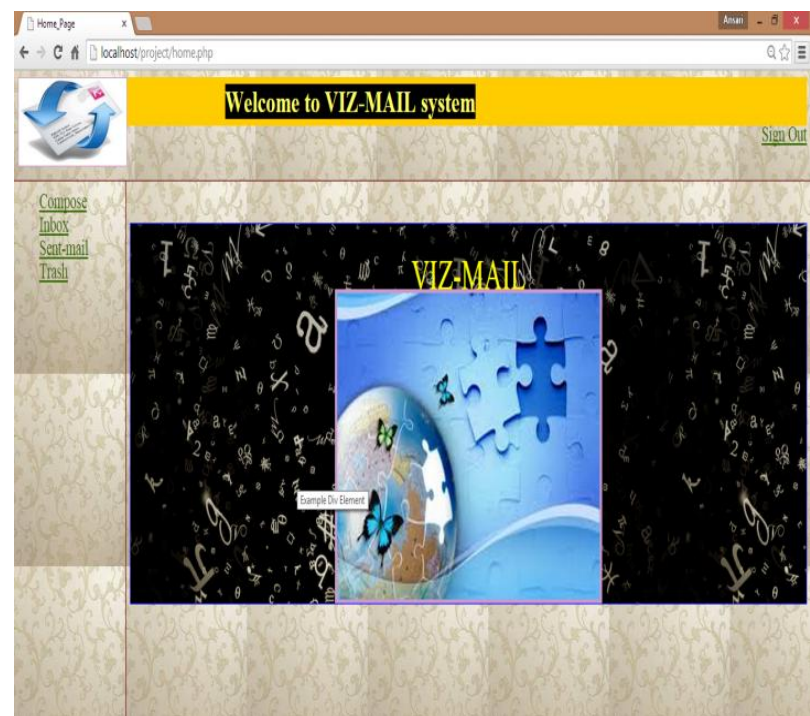

Fig. 9. Home Page

All these functionalities has been implemented. The modules given below are to be included in the system and will be implemented as a part of the proposed system. The complete walkthrough of this system is given as follows: 


\section{E. Compose mail:}

This is one of the most important options provided by the mail services. The functionality of compose mail option would not match the already existing mail system. Since the system is for visually challenged people and keyboard operations are completely avoided composing mail would only be done on voice input and mouse operations. No typed input will be required. User can directly record message that needs to be propagated and can send it. This voice massage will go in form of attachment. The receiver can hear the recording and get the message user wanted to send. User would not require attaching the file. Record option will be provided in the compose window itself. Once recorded it will confirm whether the recording is perfect or not by letting the user hear it and if the user confirms it will be automatically attached to the mail.

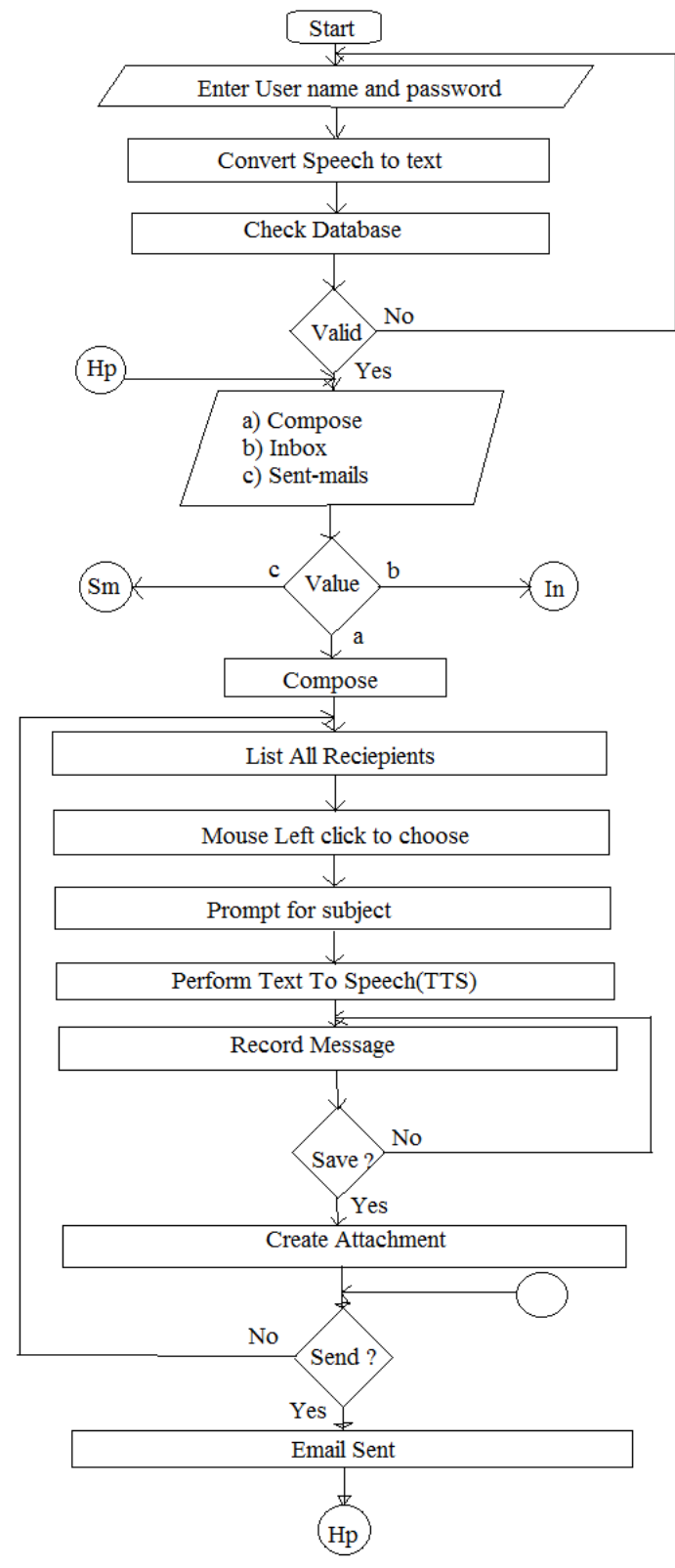

Fig. 10. Flow chart of Compose-Mail

\section{F. Inbox:}

This option helps the user view all the mails that has been received to his/her account. The user can listen to mails he/she wants to by performing the click operation specified by the prompt. In order to navigate through different mails prompt will specify which operations to perform. Each time the mail is selected the user will be prompted as whom the sender is and what is the subject of that particular mail. Accordingly user can decide whether the mail needs to be read or not or it should be deleted. Deleted mails will be saved in trash section.

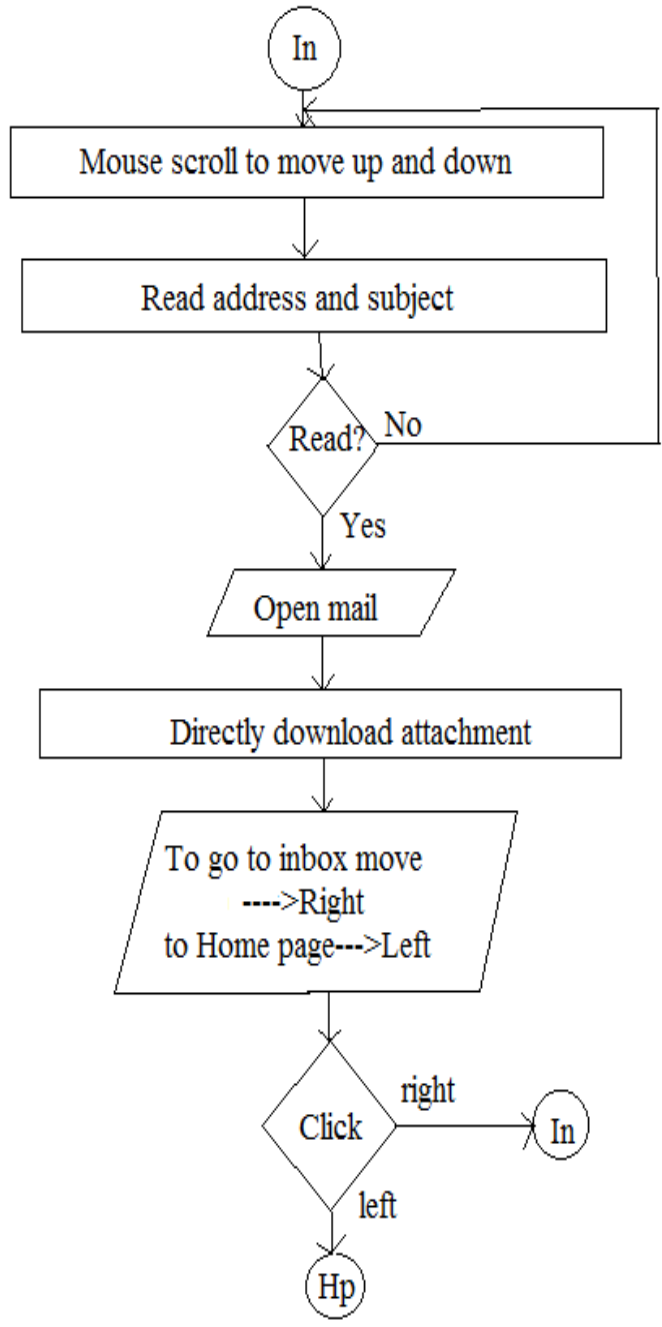

Fig. 11. Flow chart of Inbox

G. Sent mail:

This option will keep a track of all the mails sent by the user. If the user wants to access these mails, this option will provide them with their needs. In order to access the sent mails user will need to perform the actions provided by the prompt to navigate between mails. When the control lands on particular mail user will be prompted as who the receiver was and what is the subject of the mail. This will help the user in efficiently understanding and extracting the required mail. 


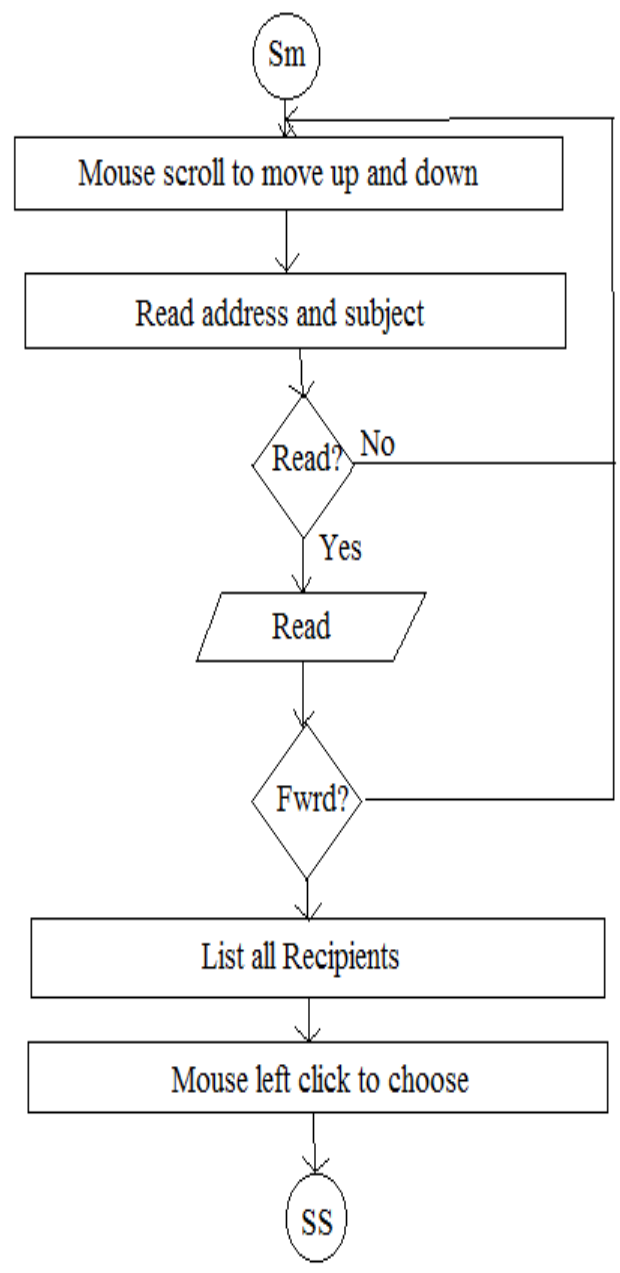

Fig. 12. Flow chart of Sent-Mail

\section{H. Trash:}

This option will keep a track of all the mails deleted by the user. Deleted mails could be the ones from inbox or sent mail. If at any time the user needs to retrieve a mail which was deleted it can be done from this option.

\section{CONCLUSION}

In this paper we have proposed a system which will help the visually impaired people to access email services efficiently. This system will help in overcoming some drawbacks that were earlier faced by the blind people in accessing emails. We have eliminated the concept of using keyboard shortcuts along with screen readers which will help reducing the cognitive load of remembering keyboard shortcuts. Also any naive user who does not know the location of keys on the keyboard need not worry as keyboard usage is eliminated. The user only needs to follow the instructions given by the IVR and use mouse clicks accordingly to get the respective services offered. Other than this the user might need to feed in information through voice inputs when specified.

\section{REFERENCES}

[1] Jagtap Nilesh, Pawan Alai, Chavhan Swapnil and Bendre M.R.. "Voice Based System in Desktop and Mobile Devices for Blind People". In International Journal of Emerging Technology and Advanced Engineering (IJETAE), 2014 on Pages 404-407 (Volume 4, issue 2)

[2] Ummuhanysifa U.,Nizar Banu P K , "Voice Based Search Engine and Web page Reader". In Internationa Journal of Computational Engineering Research (IJCER). Pages 1-5.

[3] G. Shoba, G. Anusha, V. Jeevitha, R. Shanmathi. "AN Interactive Email for Visually Impaired". In International Journal of Advanced Research in Computer and Communication Engineering (IJARCCE), 2014 on Pages 5089-5092.(Volume 3, Issue 1).

[4] The Radicati website. [Online]. Available: http://www.radicati.com/wp/wp-content/uploads/2014/01/EmailStatistics-Report-2014-2018-Executive-Summary.pdf.

[5] The WHO website. [Online]. Available: http://www.who.int/mediacentre/factsheets/fs282/en/ . 